ULB-TH/06-26

\title{
A Special Class of Rank 10 and 11 Coxeter Groups
}

\author{
Marc Henneaux $\diamond$, Mauricio Leston $₫$, Daniel Persson $\bowtie$ and Philippe Spindel ${ }^{\diamond}$ \\ - Physique Théorique et Mathématique, \\ Université Libre de Bruxelles and International Solvay Institutes, \\ Boulevard du Triomphe, ULB - C.P. 231, B-1050 Bruxelles, Belgium \\ $\diamond$ Centro de Estudios Científicos (CECS), Casilla 1469, Valdivia, Chile \\ $\triangle$ Theoretische Naturkunde, Vrije Universiteit Brussel and \\ International Solvay Institutes, Pleinlaan 2, B-1050 Brussels, Belgium \\ * Instituto de Astronomica y Fisica del Espacio (IAFE), \\ Casilla de Correo 67, Sucursal 28, 1428 Buenos Aires, Argentina \\ $\odot$ Service de Mécanique et Gravitation, \\ Université de Mons-Hainaut, Académie Wallonie-Bruxelles, \\ Avenue du Champ de Mars 6, 7000 Mons, Belgium \\ E-mail: henneaux@ulb.ac.be,mauricio@iafe.uba.ar, dpersson@ulb.ac.be, \\ Philippe.Spindel@umh.ac.be
}

\begin{abstract}
In the course of investigating regular subalgebras of $E_{10(10)}$ related to cosmological solutions of 11-dimensional supergravity supporting an electric 4 -form field, a class of rank 10 Coxeter subgroups of the Weyl group of $E_{10(10)}$ was uncovered (hep-th/0606123). These Coxeter groups all share the property that their Coxeter graphs have incidence index 3, i.e. that each node is incident to three and only three single lines. Furthermore, the Coxeter exponents are either 2 or 3 , but never $\infty$. We here go beyond subgroups of the Weyl group of $E_{10(10)}$ and classify all rank 10 Coxeter graphs with these properties. We find 21 distinct Coxeter groups of which 7 were already described in hep-th/0606123. Moreover, we extend the classification to the rank 11 case and we find 252 inequivalent rank 11 Coxeter groups with incidence index 4 , of which at least 28 can be regularly embedded into $E_{11(11)}$.
\end{abstract}

\footnotetext{
${ }^{\dagger}$ This version contains an Erratum, correcting a mistake regarding the number of symmetric rank 11 Coxeter graphs with incidence index 4. The bulk of the paper is unchanged, except for scattered comments drawing the reader's attention to where the Erratum applies.
} 


\section{Introduction}

The study of hidden symmetries of supergravity theories has revealed an intriguing connection with the theory of infinite dimensional Kac-Moody algebras. This correspondence has been most extensively explored from various directions in the context of eleven dimensional supergravity. In particular, the theory exhibits an exceptional coset symmetry $\mathcal{E}_{d(d)} / \mathcal{K}\left(\mathcal{E}_{d(d)}\right)$ when compactified on a $d$-torus $T^{d}$ (see e.g. [1] [2]). This symmetry combines the non-perturbative S-duality and the perturbative T-duality of string theory into the socalled U-duality group $\mathcal{E}_{d(d)}$. It has been conjectured that the discrete subgroup $\mathcal{E}_{d(d)}(\mathbb{Z})$ lifts to a symmetry of the full string theory [3] [4].

The appearance of these symmetries is well established for compactification down to $D=2+1$ dimensions where the U-duality symmetry is described by the split form of the largest of the exceptional Lie groups, namely $\mathcal{E}_{8(8)}$. It is furthermore known that in $D=1+1$ dimensions there is an affine symmetry group leaving the equations of motion invariant. This group is the analogue of the Geroch group and has been identified with the affine extension of $\mathcal{E}_{8(8)}$, denoted $\mathcal{E}_{9(9)} \equiv \mathcal{E}_{8(8)}^{+}$. The affine symmetry is responsible for the emergence of an integrable structure of $N=16$ supergravity reduced to 2 dimensions [5] 6] [7.

Further compactification on $T^{10}$ and $T^{11}$ is much less understood but it was conjectured long ago by Julia [8] that the chain of extended symmetries should somehow remain unbroken, thus giving rise to $\mathcal{E}_{10(10)}$ and $\mathcal{E}_{11(11)}$ as symmetry groups of eleven dimensional supergravity appropriately compactified to $D=1$ and $D=0$ dimensions, respectively.

Subsequently, West showed 9] 10] that eleven dimensional supergravity could be reformulated as a non-linear realization based on a finite dimensional Lie algebra called $G_{11}$ whose structure corresponded in part to the low-level structure of the infinite dimensional Kac-Moody algebra $E_{11(11)}=\operatorname{Lie}\left[\mathcal{E}_{11(11)}\right]$. This led him to conjecture that $E_{11(11)}$ should in fact be a symmetry of the full (uncompactified) eleven dimensional supergravity, or even of M-theory itself.

Another window into the Kac-Moody structure of M-theory was later opened through the study of eleven dimensional supergravity close to a spacelike singularity. It was found that the effective dynamics at each spatial point could be mapped onto a piecewise linear particle motion in an auxiliary space of Lorentzian signature. The piecewise nature of the motion is due to reflections against hyperplanes in the Lorentzian space and these reflections form a Coxeter group which could be identified with the Weyl group of $E_{10(10)}=\operatorname{Lie}\left[\mathcal{E}_{10(10)}\right]$ [11] [12]. This unexpected result was taken to be a strong indication that the $E_{10(10)^{-}}$ symmetry should play a fundamental role in the ultimate formulation of M-theory.

The relation between $E_{10(10)}$ and M-theory was pushed even further in [13] where a Lagrangian based on the coset space $\mathcal{E}_{10(10)} / \mathcal{K}\left(\mathcal{E}_{10(10)}\right)$ was explicitly constructed and whose dynamics reproduced the dynamics of a certain regime of eleven dimensional supergravity. This conjectural and somewhat mysterious relation between the dynamics of M-theory and 
the geodesic flow on $\mathcal{E}_{10(10)} / \mathcal{K}\left(\mathcal{E}_{10(10)}\right)$ has up until now been thoroughly tested and verified only within consistently truncated versions of both theories.

By a consistent truncation we mean a truncation such that a solution to the truncated equations of motion is also a solution to the equations of motion of the full theory. A natural truncation from the algebraic point of view is the level truncation. The level provides a grading of $E_{10(10)}$ and one may consistently truncate the theory to any finite level by setting all higher level "covariant derivatives" to zero [13]. Another useful type of truncation is that of a subgroup truncation. One restricts the dynamics on the coset space to an appropriately chosen subgroup, say $\overline{\mathcal{G}} \subset \mathcal{E}_{10(10)}$. The equations of motion imply that the evolution of initial data in $\overline{\mathcal{G}}$ remain in $\overline{\mathcal{G}}$. As long as we restrict to subgroups generated by fields that live within the established region of compatibility with the supergravity dynamics we know that solutions of the equations of motion for the sigma model $\overline{\mathcal{G}} / \mathcal{K}(\overline{\mathcal{G}})$ also correspond to exact solutions on the supergravity side.

In the cosmological regime one usually imposes conditions on the metric and the 4form field strength in order to simplify the dynamics. A class of electric solutions to 11dimensional supergravity was found long ago in [14] by considering a diagonal spatial metric and a diagonal energy momentum tensor of the 4-form. An interesting subclass of this class of solutions can be encoded in so-called geometric configurations $\left(n_{m}, g_{3}\right)$. These consist of $n$ points and $g$ lines, drawn on the plane with the following rules; (i) each line is incident to three points, (ii) each point is incident to $m$ lines and (iii) two points determine at most one line. One then associates a non-vanishing component of the electric field, $F_{0 i j k}$, for each line in the configuration and one takes a zero magnetic field. The third condition above encodes the diagonality of the energy-momentum tensor of $F_{\mu \nu \rho \sigma}$.

Cosmological solutions to eleven-dimensional supergravity were recently reinvestigated in 15 from the point of view of the proposed connection between M-theory and $E_{10(10)}$. This was further pursued by the present authors in [16] where it was found that each geometric configuration discussed in [14] corresponds in fact to a regular subalgebra, $\overline{\mathfrak{g}}$, of $E_{10(10)}$ and that the solutions of the corresponding sigma model, i.e. truncated to the relevant regular subalgebra, generalize the cosmological solutions described in [14. Some of these solutions correspond to so-called S-brane solutions and it was noted that the geometric configurations provide information about the intersection rules between S-branes. 1 . This result is in spirit very similar to the analysis of [21], where brane solutions were analyzed in the context of very extended Kac-Moody algebras.

The Dynkin diagram $\mathbb{D}_{\overline{\mathfrak{g}}}$ derived from a certain configuration $\left(n_{m}, g_{3}\right)$ is the lineincidence diagram of $\left(n_{m}, g_{3}\right)$, meaning that each line in $\left(n_{m}, g_{3}\right)$ defines a node in $\mathbb{D}_{\overline{\mathfrak{g}}}$ and two nodes in $\mathbb{D}_{\overline{\mathfrak{g}}}$ are connected only if the corresponding lines in $\left(n_{m}, g_{3}\right)$ are parallel. In particular, a set of six rank 10 Lorentzian subalgebras of $E_{10(10)}$ was uncovered in this way from the configurations with 10 points and 10 lines, $\left(n_{m}, g_{3}\right)=\left(10_{3}, 10_{3}\right)$. As a

\footnotetext{
${ }^{1}$ See [17] 18] for the original analysis of intersection rules for $p$ - and S-branes. See also [19] 20] for related discussions in the context of hyperbolic Kac-Moody algebras.
} 
consequence of the rules for constructing the configurations, the Dynkin diagrams of these algebras displayed a remarkable regularity: all nodes in the diagrams are connected to three and only three other nodes, or, in other words, each node in the Dynkin diagrams is incident to three single lines. Furthermore, the Cartan indices (off-diagonal entries of the corresponding Cartan matrix, which is symmetric in the present context) are equal to 0 or -1 and do not take lower negative values $(-2,-3, \cdots)$ even though these are in principle allowed within $E_{10(10)}$ (as pointed out in the next section).

Since none of these algebras are hyperbolic, the corresponding gravitational dynamics is non-chaotic and, in the BKL-limit, the solution settles asymptotically into a Kasner solution after finitely many oscillations [22, 23].

In the billiard analysis for generic solutions of 11-dimensional supergravity, one only sees the Weyl group of $E_{10(10)}$ because this is the group of reflections in the walls bounding the billiard table, i.e. reflections in the simple roots of $E_{10(10)}$. From this point of view the analysis of [16] revealed a new class of rank 10 Coxeter subgroups of the Weyl group of $E_{10}$. Because of the fact that the Dynkin diagrams of the Kac-Moody algebras derived from the configurations $\left(10_{3}, 10_{3}\right)$ are all connected and the corresponding Cartan matrices are symmetric, it follows that the associated Coxeter exponents can only be 2,3 or $\infty$ [28]. However, the value $\infty$ corresponding to the infinite dihedral group does not arise in the particular subclass analyzed in [16] since the only values of the Cartan integers are 0 or -1 . The Coxeter graphs inherit in addition the property of the Dynkin diagrams that the nodes are all incident to exactly three lines.

In fact, it was shown in [16] that in all geometric configurations, $\left(n_{m}, g_{3}\right)$, each line is parallel to a number $k$ of different lines, where $k$ depends on the configuration but not on the individual lines. This implies that the Coxeter graphs derived from such configurations have the property that each node, $P$, in the graph is connected to a number $k$ of other nodes, such that $k$ depends on the configuration but not on the node $P$ itself. Henceforth we will refer to the number $k$ as the incidence index and denote it by $\mathcal{I}$.

The purpose of this short note is to go beyond Coxeter groups determined by geometric configurations and classify all rank 10 Coxeter groups with the following two properties:

- the incidence index $\mathcal{I}$ is equal to $3, \mathcal{I}=3$;

- the Coxeter exponents $m_{i j}(i \neq j$, see below $)$ are equal to 2 or 3 .

The classification is equivalent to the classification of all symmetric rank 10 Cartan matrices with off-diagonal terms equal to 0 or -1 .

The classification is done by constructing the Cartan matrices from scratch in a step-bystep procedure. In this way we find that the rank 10 Coxeter groups come in three distinct classes:

- 9 Cartan matrices of Lorentzian signature, 6 of which correspond to geometric configurations. 
- 2 degenerate cases whose Cartan matrices both have vanishing determinants but with one negative eigenvalue each. One of these cases can be derived from a geometric configuration.

- 10 Cartan matrices with positive determinants but with signatures $\left(\left.2\right|_{-},\left.8\right|_{+}\right)$.

The same classification scheme is further pursued for the case of rank 11 Coxeter groups with the following result2:

- 71 Cartan matrices of Lorentzian signature, 15 of which correspond to geometric configurations and so can be embedded into $E_{11(11)}$.

- 5 Cartan matrices with negative determinants, all of which have signature $\left(\left.3\right|_{-},\left.8\right|_{+}\right)$.

- 9 Cartan matrices with vanishing determinants, all of which have one zero eigenvalue and one negative eigenvalue. 7 of these can be derived from geometric configurations.

- 1 Cartan matrix with vanishing determinant and with two negative and one zero eigenvalue.

- 166 Cartan matrices with positive determinants but with signatures $\left(\left.2\right|_{-},\left.9\right|_{+}\right)$.

Subsequent sections are organized as follows. We begin by briefly recalling some relevant properties of Coxeter groups and how they are realized as the Weyl groups of Kac-Moody algebras. In Section 3 we display in detail the complete classification of all rank 10 Coxeter groups with incidence index 3 and Coxeter exponents equal to 2 or 3 . Section 4 is devoted to a similar classification for the rank 11 case. Finally, we end the paper with a concluding discussion of our results and directions for future research.

Discussions of subalgebras of Kac-Moody algebras and in particular $E_{10(10)}$ along different but related lines may be found in [24, 25, 26, 27].

\section{Coxeter Groups - A Reminder}

The following section gives a very brief overview of some relevant aspects of Coxeter groups and their geometric realization. More information may be found in [28] [29].

A Coxeter group $\mathfrak{C}$ is abstractly defined in terms of generators $\sigma_{i}(i=1, \ldots, r)$ that obey the following relations

$$
\left(\sigma_{i} \sigma_{j}\right)^{m_{i j}}=1
$$

where the Coxeter exponents $m_{i j}$ are positive integers with the following properties

$$
\begin{aligned}
& m_{i j}=m_{j i} \\
& m_{i i}=1 \\
& m_{i j} \geq 2 \text { for } i \neq j .
\end{aligned}
$$

\footnotetext{
${ }^{2}$ This list is incomplete; see the Errata added on page 19.
} 
Note that $m_{i i}=1$ implies

$$
\sigma_{i}^{2}=1
$$

which explains why the Coxeter groups are called reflection groups. We focus in the sequel on the exponents $m_{i j}$ with $i \neq j$. These Coxeter exponents contain the complete information about the group $\mathfrak{C}$. It is convenient to encode this information in a diagram, called a Coxeter graph, $\mathbb{G}_{\mathfrak{C}}$. We associate a node in $\mathbb{G}_{\mathfrak{C}}$ to each reflection generator $\sigma_{i}$. The rank $r$ of $\mathfrak{C}$ is then equal to the number of nodes in $\mathbb{G}_{\mathfrak{C}}$. If $m_{i j}=2$, the generators $\sigma_{i}$ and $\sigma_{j}$ commute, in which case there is no line connecting nodes $i$ and $j$ in $\mathbb{G}_{\mathfrak{C}}$. Whenever $m_{i j}>2$ the nodes $i$ and $j$ are connected by a single line and one writes explicitly the component $m_{i j}$ over the line, except if $m_{i j}=3$ in which case the space over the line is left blank.

In order to see the connection with Weyl groups of Kac-Moody algebras it is useful to exhibit a geometric realization of $\mathfrak{C}$. This can be achieved by considering the Coxeter group as a group of linear transformations acting in a vector space, $V$, of dimension $r=$ Rank $\mathfrak{C}$. We will be interested in crystallographic Coxeter groups, which are the ones that stabilize a lattice, $L$, in $V$. This is the class of Coxeter groups that appear as Weyl groups of KacMoody algebras, and the lattice $L$ then corresponds to the root lattice of the Kac-Moody algebra in question. For crystallographic Coxeter groups, the exponents $m_{i j}$ are restricted to lie in the set $\{2,3,4,6, \infty\}$.

The action of $\mathfrak{C}$ on $V$ is explicitly given by

$$
\sigma_{i}\left(\alpha_{j}\right)=\alpha_{j}-2 \frac{B\left(\alpha_{i}, \alpha_{j}\right)}{B\left(\alpha_{i}, \alpha_{i}\right)} \alpha_{i}
$$

where $B($,$) denotes the scalar product in V$ and where $\left\{\alpha_{1}, \ldots, \alpha_{r}\right\}$ is a basis of of $V$. The form of Eq. (4) is precisely that of a Weyl reflection in the set of simple roots, $\alpha_{i}$, of some Kac-Moody algebra, $\mathfrak{g}$. The bilinear form $B($,$) is then the \mathfrak{g}$-invariant bilinear form restricted to the Cartan subalgebra. The integers

$$
2 \frac{B\left(\alpha_{i}, \alpha_{j}\right)}{B\left(\alpha_{i}, \alpha_{i}\right)}
$$

are called Cartan integers and form the off-diagonal entries of the Cartan matrix.

In the particular case where the simple roots $\alpha_{i}$ of the algebra $\mathfrak{g}$ all have the same length squared, conveniently taken to be equal to 2 , the Cartan matrix is symmetric and given by

$$
A_{i j}=B\left(\alpha_{i}, \alpha_{j}\right)
$$

The relation between the Cartan integers and the Coxeter exponents is given (in the symmetric case) by [29]

$$
\begin{array}{lll}
A_{i j}=0 & \Leftrightarrow \quad m_{i j}=2, \\
A_{i j}=-1 & \Leftrightarrow \quad m_{i j}=3, \\
A_{i j}<-1 & \Leftrightarrow \quad m_{i j}=\infty .
\end{array}
$$


For the case of connected Kac-Moody algebras with a symmetric Cartan matrix with only 2's, 0's and -1's, the Dynkin diagrams and the Coxeter graphs of the associated Weyl groups coincide. In order to see this we recall the rules for constructing the Dynkin diagram associated to a Cartan matrix $A^{i j}$. For each simple root there corresponds a node in the diagram. The nodes $i$ and $j(i \neq j)$ are connected by $\left|A^{i j}\right|$ lines. Thus for the cases under consideration we have always $\left|A^{i j}\right|=1$ or $\left|A^{i j}\right|=0$ so the Dynkin diagram indeed coincides with the Coxeter graph. Hence, in this case they carry the same information and instead of classifying all Coxeter exponents $m_{i j}$ we perform the classification of all Cartan matrices whose Dynkin diagrams have the desired structure. The result is the same.

Note that even though the Cartan matrix of $\mathfrak{g}=E_{10(10)}$ is symmetric and has only 0 and -1 off the diagonal, the Cartan matrix of regular subalgebras of $E_{10(10)}$ constructed along the lines of [24], while necessarily symmetric, might have integers $<-1$ off the diagonal. The corresponding Coxeter exponents might then be equal to infinity. This is because the scalar product of real, (non simple) positive roots of $E_{10(10)}$ might be $<-1$. For instance, even if one restricts one's attention to symmetry, electric, magnetic or gravitational roots, one finds that the scalar products are in the set $\{2,1,0,-1,-2,-3\}$ with -3 reached for the scalar products of some gravitational roots among themselves. Let us show this explicitly for a few examples.

Consider the roots, $\alpha$, in the $\beta$-space basis [12], where they are written as linear forms on the Cartan subalgebra, i.e.

$$
\alpha(\beta)=\sum_{i=1}^{10} \alpha_{i} \beta^{i},
$$

where a general element of the Cartan subalgebra is $h=\beta^{i} \alpha_{i}^{\vee}$. The metric in the root space of $E_{10(10)}$ is Lorentzian and takes the following form

$$
\left(\alpha \mid \alpha^{\prime}\right)=\sum_{i=1}^{10} \alpha_{i} \alpha_{i}^{\prime}-\frac{1}{9}\left(\sum_{i=1}^{10} \alpha_{i}\right)\left(\sum_{j=1}^{10} \alpha_{j}^{\prime}\right) .
$$

To illustrate the procedure, we choose arbitrarily two magnetic roots (level 2) of the form

$$
\alpha_{1}^{M}(\beta)=\beta^{1}+\beta^{2}+\beta^{3}+\beta^{4}+\beta^{5}+\beta^{6} ; \quad \alpha_{2}^{M}(\beta)=\beta^{1}+\beta^{2}+\beta^{7}+\beta^{8}+\beta^{9}+\beta^{10},
$$

and using the bilinear form $(\mid$ ) one may check that the scalar product between them is

$$
\left(\alpha_{1}^{M} \mid \alpha_{2}^{M}\right)=-2
$$

By ascending to gravitational roots at level 3 one finds scalar products with lowest negative value being -3 . For example, the two roots

$$
\alpha_{1}^{G}(\beta)=2 \beta^{1}+\beta^{2}+\cdots+\beta^{8} \quad \text { and } \quad \alpha_{2}^{G}(\beta)=\beta^{2}+\cdots+\beta^{7}+2 \beta^{9}+\beta^{10},
$$

are real and their scalar product is

$$
\left(\alpha_{1}^{G} \mid \alpha_{2}^{G}\right)=-3 .
$$


The same procedure can be applied for real roots at any level yielding scalar products taking lower and lower negative integer numbers.

In principle one can obtain arbitrarily negative scalar products by choosing real roots of higher and higher level. Coxeter subgroups of the Weyl group of $E_{10(10)}$ with Coxeter exponents equal to 2 or 3 (and not $\infty$ ) are thus rather special.

\section{Classification of Rank 10 Coxeter Groups with $\mathcal{I}=3$}

Let $\mathbb{G}_{\mathfrak{C}}$ be a rank 10 Coxeter graph with $\mathcal{I}=3$ and $m_{i j}=2,3(i \neq j)$. The structure of $\mathbb{G}_{\mathfrak{C}}$ is completely encoded in its associated Cartan matrix, $A_{\mathbb{G} \mathfrak{C}}$, which is a $10 \times 10$ symmetric matrix with 2 on each diagonal entry and zeroes on the off-diagonal except for exactly three entries in each row (and column) which are equal to -1. If an off-diagonal entry is non-vanishing, the corresponding nodes in $\mathbb{G}_{\mathfrak{C}}$ are connected by a single line. Similarly, a vanishing off-diagonal entry in $A_{\mathbb{G}_{\mathfrak{C}}}$ implies that the associated nodes in $\mathbb{G}_{\mathfrak{C}}$ are disconnected. Our task is now to find all inequivalent such matrices. We shall not give the details of the computations here but shall only outline the derivation.

We construct the matrices row by row as follows. A choice has to be made for the first row but any choice fulfilling the aforementioned requirements is acceptable. We denote a general Cartan matrix by $C$ and take without generality the first row to be

$$
\left[C_{1 i}\right]=\left(\begin{array}{llllllllll}
2 & -1 & -1 & -1 & 0 & 0 & 0 & 0 & 0 & 0
\end{array}\right),
$$

where $i=1, \ldots, 10$. Because $C$ is symmetric the next row must start as

$$
\left[C_{2 i}\right]=\left(\begin{array}{lll}
-1 & 2 & \ldots
\end{array}\right)
$$

and we know that two more entries must be equal to -1 . There are only three distinct choices. To understand this it is helpful to keep the Coxeter graph in mind. The first row tells us that node 1 is connected to nodes 2,3 and 4. As we take the next step we want to determine the number of distinct connections to node 2 . The possibilities are: (i) node 2 is further connected to 3 and 4 , (ii) node 2 is connected to 3 or 4 and then to any of the nodes in the set $\{5, \ldots, 10\}$, (iii) node 2 is connected to two of the nodes in the set $\{5, \ldots, 10\}$. Hence, we arrive at

$$
\begin{aligned}
{\left[C_{2 i}\right]_{(\mathbf{1})} } & =\left(\begin{array}{rrrrrrrrrr}
-1 & 2 & -1 & -1 & 0 & 0 & 0 & 0 & 0 & 0
\end{array}\right) \\
{\left[C_{2 i}\right]_{(\mathbf{2})} } & =\left(\begin{array}{llllllllll}
-1 & 2 & -1 & 0 & -1 & 0 & 0 & 0 & 0 & 0
\end{array}\right) \\
{\left[C_{2 i}\right]_{(\mathbf{3})} } & =\left(\begin{array}{llllllllll}
-1 & 2 & 0 & 0 & -1 & -1 & 0 & 0 & 0 & 0
\end{array}\right) .
\end{aligned}
$$

We now proceed to the third row. For $C_{(\mathbf{1})}$ and $C_{(\mathbf{2})}$, the off-diagonal components $\{3,1\}$ and $\{3,2\}$ are already fixed to -1 by symmetry. We must find the inequivalent ways to add the third non-vanishing entry. $C_{(\mathbf{1})}$ admits two possibilities: $\left[C_{34}\right]_{(\mathbf{1})}=-1$ or $\left[C_{35}\right]_{(\mathbf{1})}=-1$. For $C_{(\mathbf{2})}$, there are three distinct choices for the entry -1 , namely $\left[C_{34}\right]_{(\mathbf{2})},\left[C_{35}\right]_{(\mathbf{2})}$ or $\left[C_{36}\right]_{(\mathbf{2})}$. 
In the third case, $C_{(\mathbf{3})}$, only the first entry of the third row is determined by symmetry. This gives us five different possibilities for the distribution of two non-vanishing off-diagonal entries:

$$
\begin{aligned}
& {\left[C_{3 i}\right]_{(3)_{1}}=\left(\begin{array}{llllllllll}
-1 & 0 & 2 & -1 & -1 & 0 & 0 & 0 & 0 & 0
\end{array}\right)} \\
& {\left[C_{3 i}\right]_{(\mathbf{3})_{2}}=\left(\begin{array}{llllllllll}
-1 & 0 & 2 & -1 & 0 & 0 & -1 & 0 & 0 & 0
\end{array}\right)} \\
& {\left[C_{3 i}\right]_{(\mathbf{3})_{3}}=\left(\begin{array}{llllllllll}
-1 & 0 & 2 & 0 & -1 & -1 & 0 & 0 & 0 & 0
\end{array}\right)} \\
& {\left[C_{3 i}\right]_{(\mathbf{3})_{4}}=\left(\begin{array}{llllllllll}
-1 & 0 & 2 & 0 & -1 & 0 & -1 & 0 & 0 & 0
\end{array}\right)} \\
& {\left[C_{3 i}\right]_{(3)_{5}}=\left(\begin{array}{llllllllll}
-1 & 0 & 2 & 0 & 0 & 0 & -1 & -1 & 0 & 0
\end{array}\right) \text {. }}
\end{aligned}
$$

At this point we thus have ten different cases to consider. Repeating the same procedure we find 33 possibilities after adding the fourth row, 98 possibilities after the fifth row, 296 after the sixth and 574 possibilities after the seventh row.

The remaining task is to add rows eight, nine and ten. These last steps will actually considerably restrict the number of distinct possibilities because many of the matrices we have found so far cannot be extended up to rank 10 in such a way as to preserve the condition $\mathcal{I}=3$ : the construction might get obstructed. First of all, we know that the tenth row will be completely fixed by symmetry. Hence, we must find the various possibilities for rows eight and nine. Only the entries $\left[C_{89}\right],\left[C_{8(10)}\right]$ and $\left[C_{9(10)}\right]$ are undetermined by symmetry. To this end we consider the triple $\left(s_{8}, s_{9}, s_{10}\right)$, where $s_{8}, s_{9}$ and $s_{10}$ denote the three sums

$$
s_{k}=\sum_{i=1}^{7}\left|\left[C_{k i}\right]\right| \quad k=8,9,10 .
$$

The various possibilities for the values of the triple, $\left(s_{8}, s_{9}, s_{10}\right)$, fixes the distinct choice for the remaining entries $\left[C_{89}\right],\left[C_{8(10)}\right]$ and $\left[C_{9(10)}\right]$ according to

$\begin{array}{cccrrr}s_{8} & s_{9} & s_{10} & {\left[C_{98}\right]} & {\left[C_{8(10)}\right]} & {\left[C_{9(10)}\right]} \\ 1 & 1 & 1 & -1 & -1 & -1 \\ 1 & 2 & 2 & -1 & -1 & 0 \\ 2 & 1 & 2 & -1 & 0 & -1 \\ 2 & 2 & 1 & 0 & -1 & -1 \\ 2 & 2 & 3 & -1 & 0 & 0 \\ 2 & 3 & 2 & 0 & -1 & 0 \\ 3 & 2 & 2 & 0 & 0 & -1 \\ 3 & 3 & 3 & 0 & 0 & 0 .\end{array}$

All other choices of $\left(s_{8}, s_{9}, s_{10}\right)$ are incompatible with having incidence index 3 . For instance the first two 1's in the triple $(1,1,2)$ forces $\left[C_{89}\right]=\left[C_{8(10)}\right]=\left[C_{9(10)}\right]=-1$ with the resulting contradiction that there are four -1 's on the last line. Therefore, out of the 574 Cartan matrices we had up to seven rows, we find that only 256 of them allow for an extension to 
rank 10 in accordance with Eq. (26). Among these $10 \times 10$ matrices, 109 are Lorentzian (i.e. have negative determinant), 12 are degenerate (one null, one negative and eight positive eigenvalues) and 135 of them have positive determinant but come with signature $\left(\left.2\right|_{-},\left.8\right|_{+}\right)$.

The final step consists of determining how many of these matrices are actually equivalent up to a permutation of the vertices in the associated Coxeter graphs, or, in other words, up to a simultaneous exchange of rows and columns so as to preserve the occurrence of 2 on the diagonal. We have done this explicitly and found that many of the matrices obtained so far were in fact equivalent. We obtained that in addition to the 6 Lorentzian cases discovered in [16] there are only 3 distinct Cartan matrices with Lorentzian signature. Furthermore, in [16] we uncovered one case which was degenerate and it turns out that only one additional case exists. Finally, out of the 135 matrices with positive determinants, 10 are distinct (and have signature with $8+$ 's and 2 -'s). The result is thus that there exist 21 rank 10 Coxeter groups, or, equivalently, 21 rank 10 indefinite Kac-Moody algebras, with incidence index 3. The corresponding Coxeter graphs are reproduced in Tables $1-4$.

\section{Classification of Rank 11 Coxeter Groups with $\mathcal{I}=4$}

The particular class of rank 10 Coxeter groups discussed above were discovered by examining geometric configurations of the type $\left(n_{m}, g_{3}\right)=\left(10_{3}, 10_{3}\right)$. A similar analysis can be done for the geometric configurations with 11 points and 11 lines, $\left(n_{m}, g_{3}\right)=\left(11_{3}, 11_{3}\right)$. There exist 31 such configurations [30] and each of these gives rise, through its line-incidence diagram, to a Coxeter subgroup of the Weyl group of $E_{11(11)}$, whose significance in the context of M-theory was first pointed out in [10]. The procedure is identical to the one performed for the $\left(10_{3}, 10_{3}\right)$-configurations in [16]. This is easy to understand given the fact that the field contents for $E_{10(10)}$ and $E_{11(11)}$ at low levels are the same. All $\left(11_{3}, 11_{3}\right)$-configurations have the property that each line is parallel to four other lines and hence the associated Coxeter graphs have incidence index $\mathcal{I}=4$. Moreover the Coxeter exponents lie in the set $\{2,3\}$.

Following the procedure outlined in Section 3 we have classified all rank 11 Coxeter groups with these properties. The end result is that there exist 252 rank 11 Coxeter groups with $\mathcal{I}=4$. Of these, 28 can be obtained from geometric configurations and so correspond to subgroups of the Weyl group of $E_{11(11)}$.

Some new features arise in comparison to the rank 10 case: (i) also Cartan matrices with non-vanishing determinants come with a degeneracy, (ii) one of the Cartan matrices with vanishing determinant have two negative eigenvalues, (iii) some of the Cartan matrices with negative determinants have signatures $\left(\left.3\right|_{-},\left.8\right|_{+}\right)$. The various classes of Cartan matrices were listed in Section 1 so we will not repeat it here.

Because of obvious restrictions of space we do not exhibit the full list of rank 11 Coxeter graphs. However, in Table 5 we display some selected graphs that do not arise as lineincidence diagrams of geometric configurations. All the 252 Cartan matrices are assembled in the file "Coxeter11-4.nb" which is included in "Coxeter.zip" that can be downloaded 
from the database arXiv.org of Cornell University [33. 3

\section{Conclusions}

In this note we have extended the results of [16] by classifying all rank 10 Coxeter groups with incidence index $\mathcal{I}=3$ and Coxeter exponents $m_{i j}=2,3(i \neq j)$, including those that are not determined by geometric configurations. We find that except for the 7 cases that were uncovered in [16], there exist 14 additional Coxeter groups with similar properties. Among those, 10 cannot be regularly embedded in $E_{10(10)}$ since they do not have the correct signature. Although we do not know if the other Coxeter groups correspond to subgroups of the Weyl group of $E_{10(10)}$, one might speculate that they can perhaps be associated with some other class of subalgebras of $E_{10(10)}$ that goes beyond the "electric" subalgebras previously considered.

The natural starting point for such an investigation would be to ascend to level 2 in the decomposition of $E_{10(10)}$ and investigate "magnetic" subalgebras. These correspond to geometric configurations of the type $\left(n_{m}, g_{6}\right)$, i.e. with 6 points on each line. It is possible that some of the rank 10 Coxeter groups derived in this way could have $\mathcal{I}=3$ and Coxeter exponents $m_{i j}=2,3$ and would thus fall into the classification scheme of this paper.

We have also classified all rank 11 Coxeter groups with incidence index $\mathcal{I}=4$. The analysis revealed 252 such Coxeter groups, including 28 that can be obtained from $\left(11_{3}, 11_{3}\right)$ configurations and so can be regularly embedded in $E_{11(11)}$.

We should mention here the following related fact. Because the exponent $\infty$ does not occur among the Coxeter exponents, any pair of elements in the set of generating reflections generates a finite group (the group is called "2-spherical" [32]). 2-spherical Coxeter subgroups of $E_{10(10)}$ and $E_{11(11)}$ are rather rare - in fact, up to conjugation, there are only a finite number of them [32]. It would be of interest to determine them all.

Acknowledgments: $\mathrm{MH}$ is grateful to the organizers of the "Fourth International School on Field Theory and Gravitation", held in Rio de Janeiro in May 2006, for their kind hospitality and for the opportunity to lecture on cosmological billiards. We thank PierreEmmanuel Caprace, Arjan Keurentjes and Bernhard Mühlherr for discussions at various stages in the preparation of this article. Work supported in part by IISN-Belgium (convention 4.4511.06 (M.H. and P.S) and convention 4.4505.86 (M.H. and D.P)), by the Belgian National Lottery, by the European Commission FP6 RTN programme MRTN-CT-2004005104 (M.H., M.L. and D.P.), and by the Belgian Federal Science Policy Office through the Interuniversity Attraction Pole P5/27. Mauricio Leston was also supported in part by the "FWO-Vlaanderen" through project G.0428.06 and by a CONICET graduate scholarship.

\footnotetext{
${ }^{3}$ Since the first version of this article, the file "Coxeter11-4.nb" has been replaced by "Coxeter11-4v4.nb" that contains the correct number, 266, of inequivalent rank 11 Cartan matrices with incidence index 4. See Errata on page 19.
} 


\section{References}

[1] E. Cremmer, B Julia, H. Lu, C.N. Pope, "Dualisation of Dualities I," Nucl. Phys. B 523, 73 (1998) arXiv:hep-th/9710119.

[2] E. Cremmer, B Julia, H. Lu, C.N. Pope, "Higher Dimensional Origin of $\mathrm{D}=3$ Coset Symmetries", arXiv:hep-th/9909099.

[3] C. Hull and P. Townsend, "Unity of Superstring Dualities", Nucl. Phys. B 438, 109 (1995) arXiv:hep-th/9410167.

[4] N. Obers and B. Pioline, "U-duality and M-theory," Phys. Rept. 318, 113 (1999) arXiv:hep-th/9809039.

[5] H. Nicolai, "The integrability of N=16 supergravity," Phys. Lett. B 194, 402 (1987).

[6] H. Nicolai and N. P. Warner, "The structure of N=16 supergravity in two dimensions," Commun. Math. Phys. 125, 369 (1989).

[7] H. Nicolai and H. Samtleben, "Integrability and canonical structure of $d=2, N=16$ supergravity," Nucl. Phys. B 533, 210 (1998) arXiv:hep-th/9804152.

[8] B. Julia in: Lectures in Applied Mathematics, Vol 21, AMS-SIAM, p.335, 1985; preprint LPTENS 80/16.

[9] P. West, "Hidden superconformal symmetry in M-theory," JHEP 0008, 007 (2000) arXiv:hep-th/0005270.

[10] P. West, " $E_{11}$ and M-theory", Class. Quant. Grav. 18 (2001) arXiv:hep-th/0104081.

[11] T. Damour and M. Henneaux, " $E_{10}, B E_{10}$ and arithmetical chaos in superstring cosmology," Phys. Rev. Lett. 86, 4749 (2001) arXiv:hep-th/0012172.

[12] T. Damour, M. Henneaux and H. Nicolai, "Cosmological Billiards," Class. Quant. Grav. 20, R145 (2003) arXiv:hep-th/0212256.

[13] T. Damour, M. Henneaux and H. Nicolai, " $E_{10}$ and a 'small tension expansion' of M theory," Phys. Rev. Lett. 89, 221601 (2002) arXiv:hep-th/0207267.

[14] J. Demaret, J. L. Hanquin, M. Henneaux and Ph. Spindel, "Cosmological Models In Eleven-Dimensional Supergravity," Nucl. Phys. B 252, 538 (1985).

[15] A. Kleinschmidt and H. Nicolai, " $E_{10}$ cosmology," JHEP 0601, 137 (2006) arXiv:hep-th/0511290.

[16] M. Henneaux, M. Leston, D. Persson and Ph. Spindel, "Geometric Configurations, Regular Subalgebras of $E_{10}$ and M-theory Cosmology," JHEP 10, 021 (2006) arXiv:hep-th/0606123. 
[17] R. Argurio, F. Englert and L. Houart, "Intersection rules for p-branes," Phys. Lett. B 398, 61 (1997) arXiv:hep-th/9701042.

[18] N. Ohta, "Intersection rules for S-branes," Phys. Lett. B 558, 213 (2003) arXiv:hep-th/0301095.

[19] V.D. Ivashchuk, S.W. Kim and V.N. Melnikov, "Hyperbolic Kac-Moody algebra from intersecting p-branes," J. Math. Phys. 40, 4072 (1999) arXiv:hep-th/9803006.

[20] V.D. Ivashchuk and V.N. Melnikov, "Billiard representation for multidimensional cosmology with intersecting p-branes near the singularity," J. Math. Phys. 41, 6341 (2000) arXiv:hep-th/9904077.

[21] F. Englert, L. Houart and P. West, "Intersection rules, dynamics and symmetries," JHEP 0308, 025 (2003) arXiv:hep-th/0307024;

F. Englert, and L. Houart, " $\mathcal{G}^{+++}$invariant formulation of gravity and M-theories: Exact BPS solutions", JHEP, 01, 002 (2004) arXiv:hep-th/0311255;

F. Englert, and L. Houart, " $\mathcal{G}^{+++}$invariant formulation of gravity and M-theories: Exact intersecting brane solutions", JHEP, 05, 059 (2004) arXiv:hep-th/0405082.

[22] T. Damour, M. Henneaux, B. Julia and H. Nicolai, "Hyperbolic Kac-Moody algebras and chaos in Kaluza-Klein models," Phys. Lett. B 509, 323 (2001) arXiv:hep-th/0103094.

[23] J. Demaret, J. L. Hanquin, M. Henneaux, Ph. Spindel and A. Taormina, "The Fate of the Mixmaster Behavior in Vacuum Inhomogeneous Kaluza-Klein Cosmological Models," Phys. Lett. B 175, 129 (1986).

[24] A. J. Feingold and H. Nicolai, "Subalgebras of Hyperbolic Kac-Moody Algebras," arXiv:math.qa/0303179.

[25] J. Brown, O. J. Ganor and C. Helfgott, "M-theory and $E_{10}$ : Billiards, branes, and imaginary roots," JHEP 0408, 063 (2004) arXiv:hep-th/0401053;

J. Brown, S. Ganguli, O. J. Ganor and C. Helfgott, " $E_{10}$ orbifolds," JHEP 0506, 057 (2005) arXiv:hep-th/0409037.

[26] A. Kleinschmidt, "Indefinite Kac-Moody Algebras in String Theory", PhD Thesis, Cambridge (2004).

[27] M. Bagnoud, L. Carlevaro, "Hidden Borcherds symmetries in $Z_{n}$ orbifolds of M-theory and magnetized D-branes in Type 0' orientifolds," arXiv:hep-th/0607136.

[28] J. E. Humphreys, "Reflection Groups and Coxeter Groups", Cambridge Studies in Advanced Mathematics, 1992. 
[29] V. Kac, "Infinite dimensional Lie algebras", 3rd Ed., Cambridge University Press (1990).

[30] W. Page and H. L. Dorwart, "Numerical Patterns and Geometrical Configurations", Mathematics Magazine 57, No. 2, 82-92 (1984).

[31] See the link: http://arxiv.org/abs/hep-th/0610278.

[32] P.-E. Caprace, "Conjugacy of one-ended subgroups of Coxeter groups and parallel walls", math.GR/0508057.

[33] See the link: http://arxiv.org/abs/hep-th/0610278 v4

[34] M. Henneaux, M. Leston, D. Persson and P. Spindel, "A Special Class of Rank 10 and 11 Coxeter Groups," J. Math. Phys. 48 (2007) 053512 arXiv:hep-th/0610278.

[35] See the link: http://www.mathe2.uni-bayreuth.de/markus/reggraphs.html 


Coxeter Graph $\mathbb{C}_{C}$

Table 1: Lorentzian rank 10 Coxeter graphs with incidence index $\mathcal{I}=3$ and Coxeter exponents 2 or 3 . All of these graphs correspond to line-incidence diagrams of geometric configurations of the type $\left(n_{m}, g_{3}\right)$. 


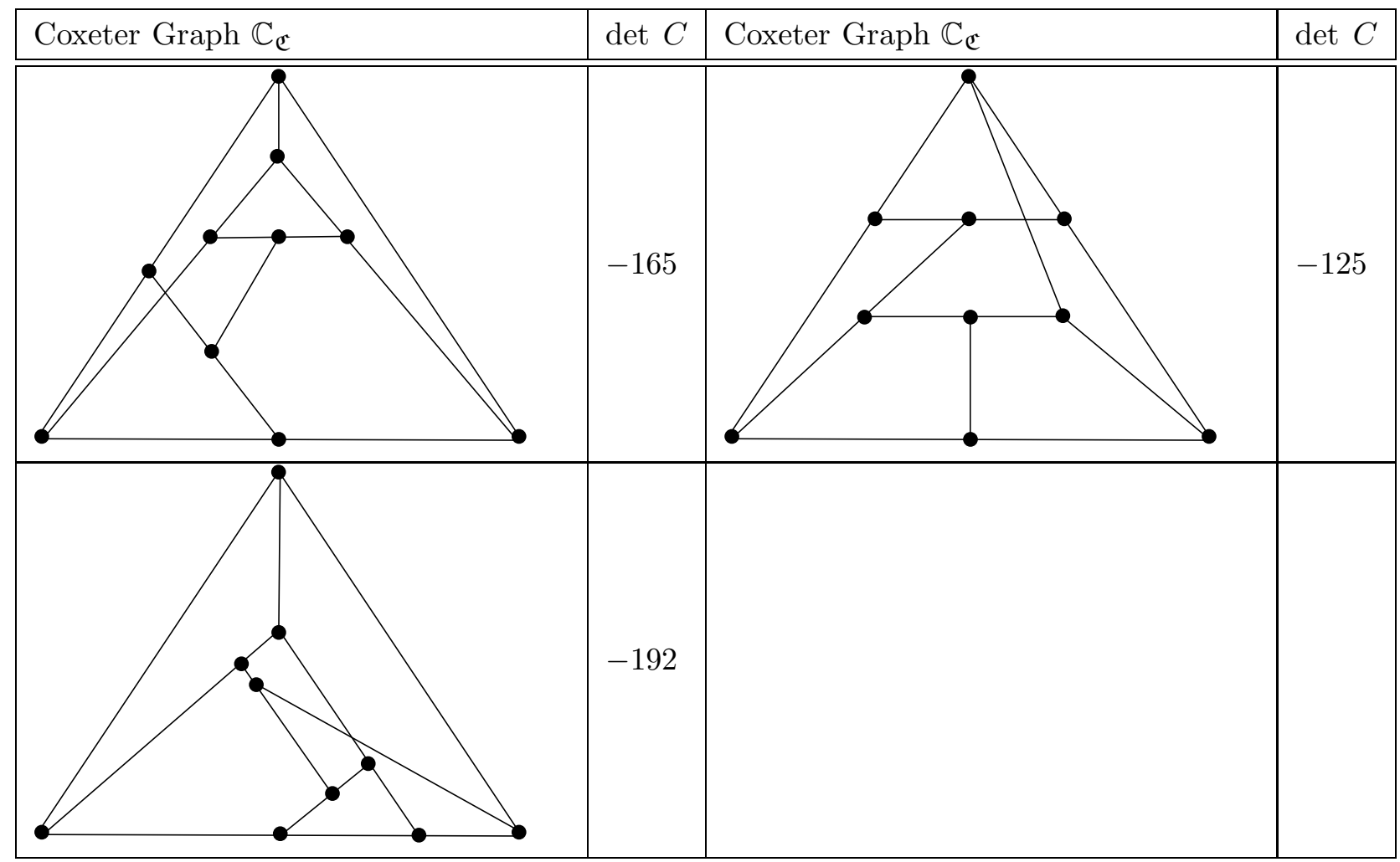

Table 2: Lorentzian rank 10 Coxeter graphs with incidence index $\mathcal{I}=3$ and Coxeter exponents 2 or 3 . None of these graphs correspond to line-incidence diagrams of geometric configurations of the type $\left(n_{m}, g_{3}\right)$.

\begin{tabular}{|l|l|l|l|}
\hline Coxeter Graph $\mathbb{C}_{\mathfrak{C}}$ & det $C$ & Coxeter Graph $\mathbb{C}_{\mathfrak{C}}$ & $\operatorname{det} C$ \\
\hline \hline & &
\end{tabular}

Table 3: Degenerate rank 10 Coxeter graphs with incidence index $\mathcal{I}=3$ and Coxeter exponents 2 or 3 . Only the leftmost graph correspond the line-incidence diagram of a geometric configuration of the type $\left(n_{m}, g_{3}\right)$. 


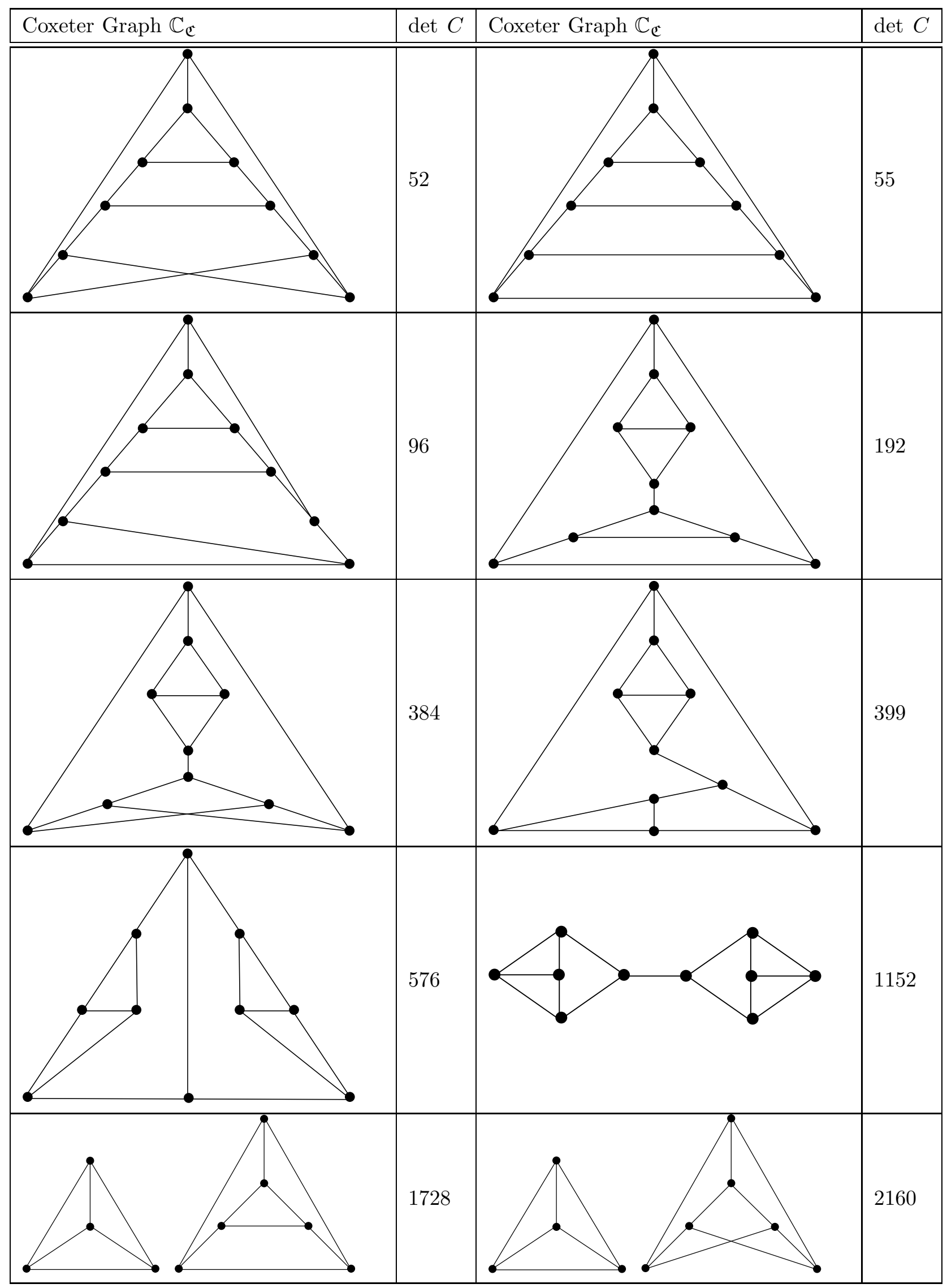

Table 4: Rank 10 Coxeter graphs with incidence index $\mathcal{I}=3$, Coxeter exponents 2 or 3 , and signature $\left(\left.2\right|_{+},\left.8\right|_{-}\right)$. None of these graphs correspond to line-incidence diagrams of geometric configurations of the type $\left(n_{m}, g_{3}\right)$. 


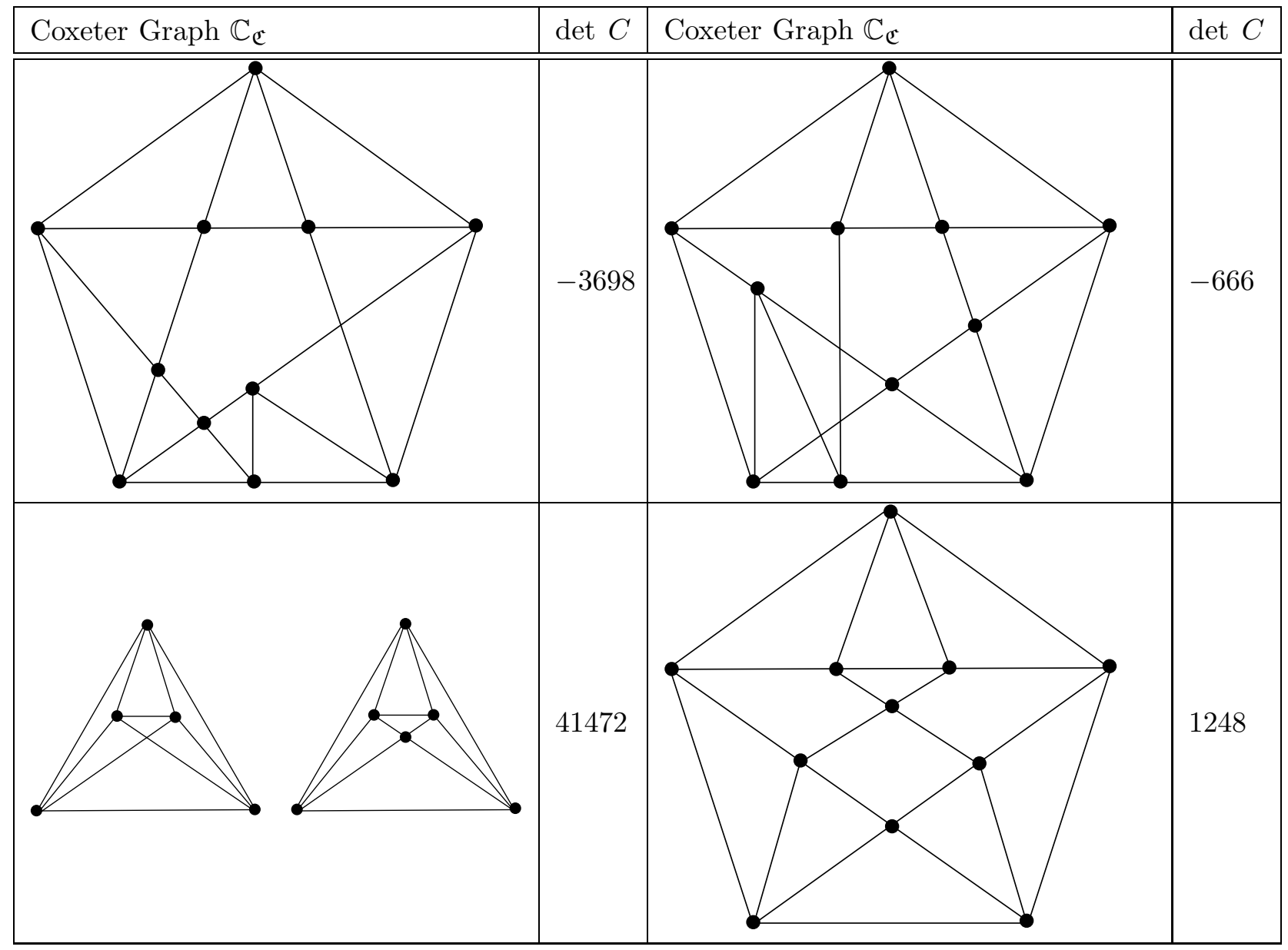

Table 5: A selection of the Rank 11 Coxeter graphs with incidence index $\mathcal{I}=4$ and Coxeter exponents equal to 2 or 3 . The top left graph is the one whose determinant is most negative and the bottom left is the one whose determinant is the most positive. None of these graphs correspond to line-incidence diagrams of geometric configurations of the type $\left(n_{m}, g_{3}\right)$. 


\section{Erratum}

In [34] we classified all rank 10 (resp. 11) Coxeter groups with incidence index $\mathcal{I}$ equal to 3 (resp. 4) and Coxeter exponents equal to 2 or 3 . This problem is equivalent to the classification of all symmetric rank 10 (resp. 11) Cartan matrices with off-diagonal components equal to 0, except for three (resp. four) on each line equal to -1 . The method we used was to first construct a redundant set of all such Cartan matrices, after which we extracted from this the subset of matrices differing by their set of eigenvalues (characteristic polynomial). In this way we obtained the 19 different rank 10 Coxeter groups, but only 252 of the 266 existing rank 11 Coxeter graphs with these properties [35]. The origin of this discrepancy is due to the inadequacy of the method adopted. Indeed, there exist distinct symmetric rank 11 Cartan matrices that have the same set of eigenvalues, but are not equivalent, in the sense that they cannot be related through conjugation by any permutation matrix.

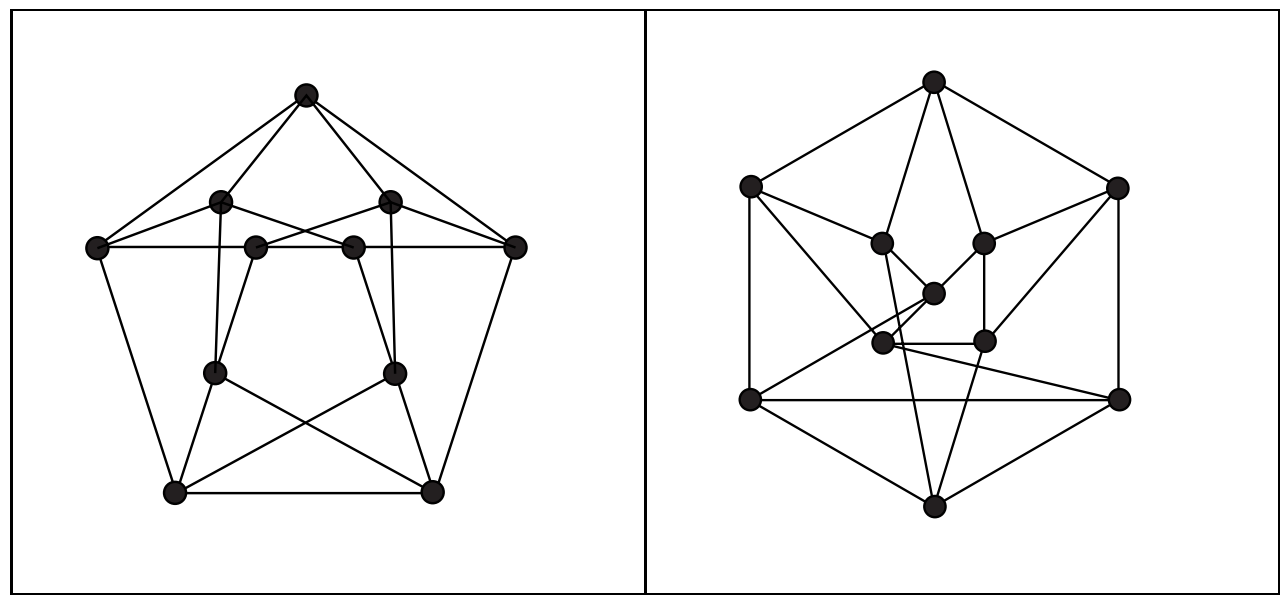

Table 6: An example of two rank 11 Coxeter graphs with incidence index $\mathcal{I}=4$. The left hand graph admits a $\mathbb{Z}_{2}$ automorphism group, while the right hand one does not admit any non-trivial automorphism. Both have the same characteristic polynomial: $-X^{11}+22 X^{10}-$ $198 X^{9}+916 X^{8}-2123 X^{7}+1088 X^{6}+6578 X^{5}-17658 X^{4}+19939 X^{3}-10988 X^{2}+2583 X-170$.

Table 6 provides an example of two such configurations, whose adjacency matrices give the same invariant polynomial, but nevertheless correspond to inequivalent Dynkin diagrams. Thus, given these considerations, the results presented in [34] have to be amended in the following way.

There exist 266 rank 11 Coxeter groups that split into the following subsets:

- 73 Cartan matrices of Lorentzian signature, 15 of which correspond to geometric configurations and so can be embedded into $E_{11(11)}$. Among them two pairs have the same eigenvalues, but are inequivalent.

- 5 Cartan matrices with negative determinants, all of which have signature $\left(\left.3\right|_{-},\left.8\right|_{+}\right)$. 
- 11 Cartan matrices with vanishing determinants, all of which have one zero eigenvalue and one negative eigenvalue. Among them three inequivalent ones have the same set of eigenvalues, and seven can be derived from geometric configurations.

- 1 Cartan matrix with vanishing determinant and with two negative and one zero eigenvalue.

- 176 Cartan matrices with positive determinants but with signatures $\left(\left.2\right|_{-},\left.9\right|_{+}\right)$. In terms of their set of eigenvalues they split into 157 singlets, 8 pairs and 1 triplet.

All the 266 inequivalent Cartan matrices are assembled in the file "Coxeter11-4v4.nb" which is included in "Coxeterv4.zip" that can be downloaded from the database arXiv.org of Cornell University [33].

Acknowledgments: We are grateful to P-E. Caprace and H. Mélot for discussions, and for providing us with useful references. 